\title{
Microstructural, Structural and Mechanical Behavior of WC-Based Hardmetals Bonded with High and Medium Entropy Alloys.
}

\author{
M.A. Ruiz-Esparza-Rodriguez ${ }^{1}$, C.G. Garay-Reyes ${ }^{1}$, I. Estrada-Guel ${ }^{1}$ and R. Martinez-Sanchez ${ }^{1}$. \\ 1. Centro de Investigación en Materiales Avanzados (CIMAV), Laboratorio Nacional de \\ Nanotecnología, Miguel de Cervantes 120, 31136 Chihuahua, Chih., México.
}

Cemented carbides are formed by hard phase and a binder, typically Tungsten Carbide (WC) and Cobalt (Co), in a proportion 80, $20 \mathrm{wt}$. \%, respectively. The Co function is join the carbide hard particles and provide toughness to the cemented carbide [1]. High entropy alloys or multicomponent systems have the characteristic of forming a single solid solution or a mixture of solid solution phases [2]. These phases resemble the common structures of FCC, BCC and HCP. In which, FCC structures tend to be very ductile, while BCC show an increase in mechanical strength. A mixture of FCC and the BCC phases take advantage of both properties, resulting in a high strength ductile material [2]. This work focuses on evaluating the microstructural, structural and mechanical behavior of high (HEA) and medium (MEA) entropy alloys as substitutes for cobalt in cemented carbides.

The HEA and MEA, CoCrFeMnNi and CoCrNi, respectively, were synthetized by mechanical alloying (MA). The elemental powders were taken with a purity of $99.99 \% .10 \mathrm{~h}$ of milling time in a high-energy mill Spex 8000 was used to fabricate MEA and HEA. Subsequently, the milling of WC-MEA and WCHEA compounds was for $2 \mathrm{~h}$ milling time with variation of the composition determined in $80,85,90$ and $95 \% \mathrm{WC}$ (wt. \%). The container is done by WC to prevent external contamination. Ar atmosphere to control the presence of oxygen during the MA process, powder mass $8.5 \mathrm{~g}$ and a ball-to-powder weight ratio of 5:1 were the process conditions used in all the experimental runs. The compaction of the powders was carried out by means of a hydraulic press under a compaction pressure of $1.56 \mathrm{GPa}$ for 5 minutes, the sintering process was maintained at $1300^{\circ} \mathrm{C}$ for $3 \mathrm{~h}$ in vacuum sealed quartz ampoules. The structural and microstructural characterization was carried out by a Panalytical X'Pert PRO x-ray diffractometer, scanning electron microscope HITACHI SU3500 and Vickers microhardness was evaluated in LM300 AT tester.

The figure 1 shows X-ray diffraction patterns obtained in WC-HEA and WC-MEA compounds, a material with a hexagonal structure (WC) and peaks corresponding to oxides in all the concentrations carried out are observed. The SEM micrographs in figure 2 shows the microstructure of WC-HEA and WC-MEA compounds, a greater oxidation (dark phase) in WC-MEA samples in comparison with the WC-HEA samples is observed. However, a greater porosity in WC-HEA samples in comparison with the WC-MEA samples is observed. The figure 3 shows the hardness values obtained in WC-HEA and WC-MEA compounds. The hardness values obtained in both compounds are close. Nevertheless, an increase gradually in function of content of WC is observed.

\section{References:}

[1] Lining G, Jiwu H, and Chen X, International Journal of Refractory Metals and Hard Materials, Volume 42 (2014), p. 228.

[2] Miraclea D.B., Senkova O.N., Acta Materialia, Volume 122 (2017), p. 448. 
a)

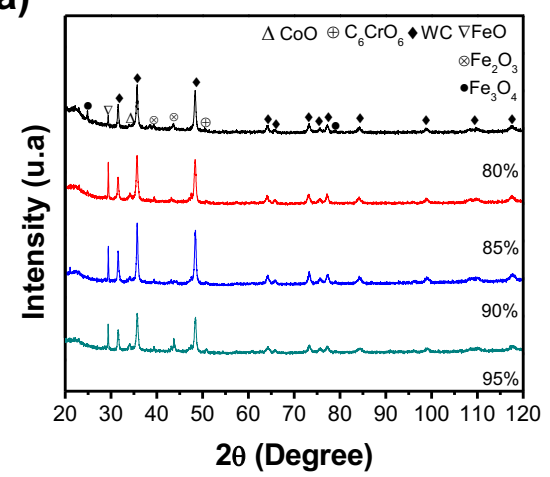

b)

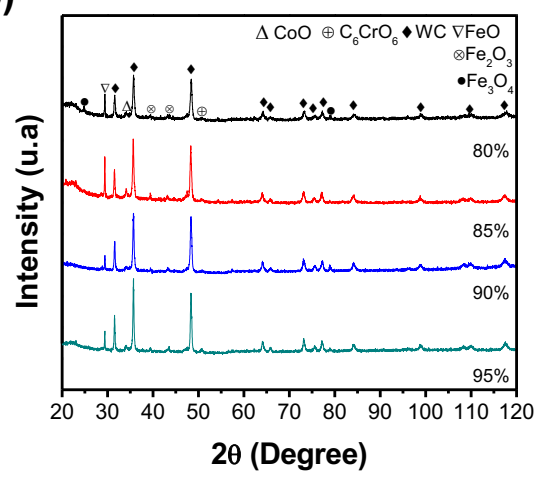

Figure 1. X-ray diffraction patterns of the sintered samples, a) WC-MEA and b) WC-HEA, with variation of the composition determined in $80,85,90$ and $95 \% \mathrm{WC}$ (wt. \%).

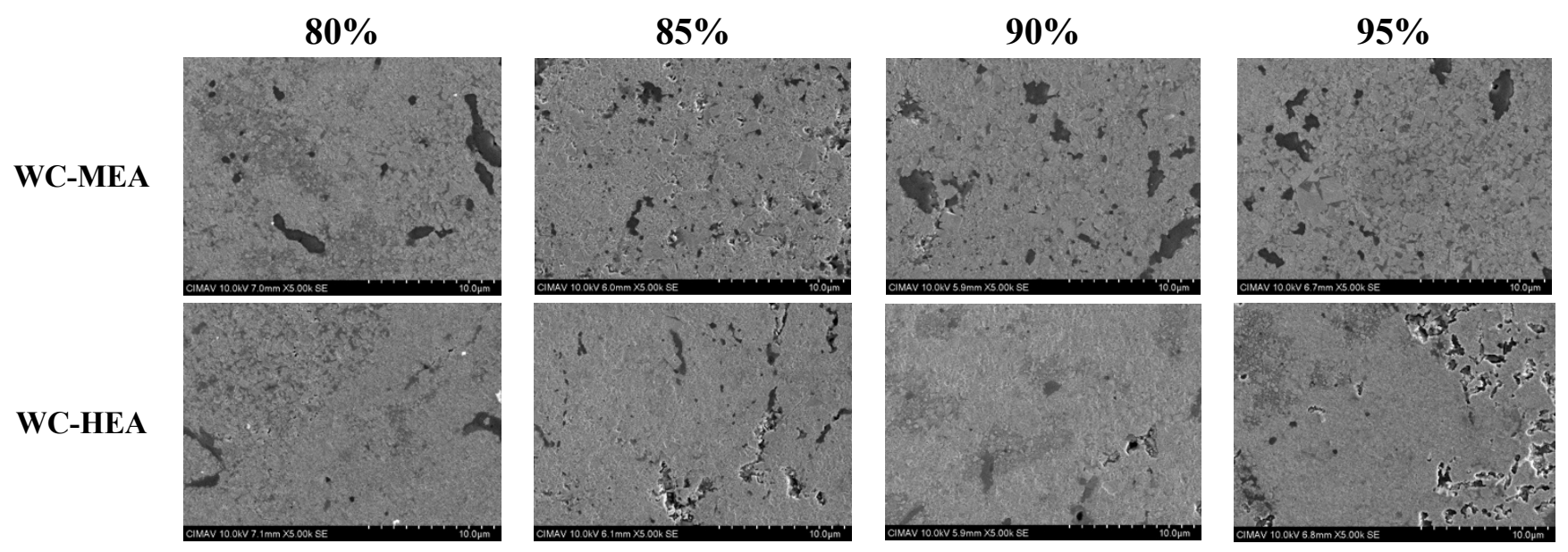

Figure 2. SEM Micrographs of the sintered samples, WC-MEA and WC-HEA, with variation of the composition determined in $80,85,90$ and $95 \% \mathrm{WC}$ (wt. \%).

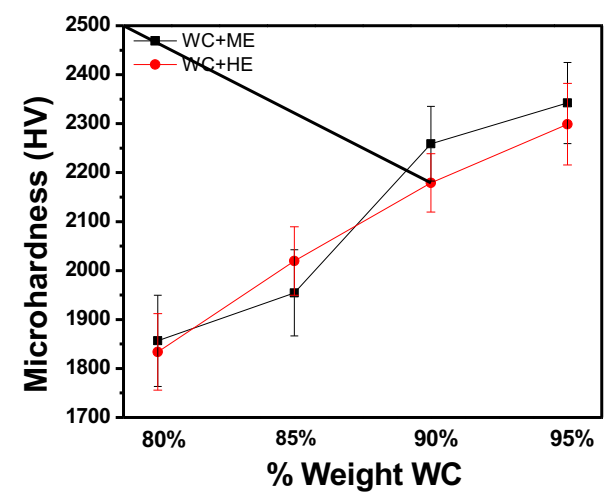

Figure 3. Vickers microhardness values of the sintered samples, WC-MEA and WC-HEA, with variation of the composition determined in $80,85,90$ and $95 \% \mathrm{WC}$ (wt. \%). 